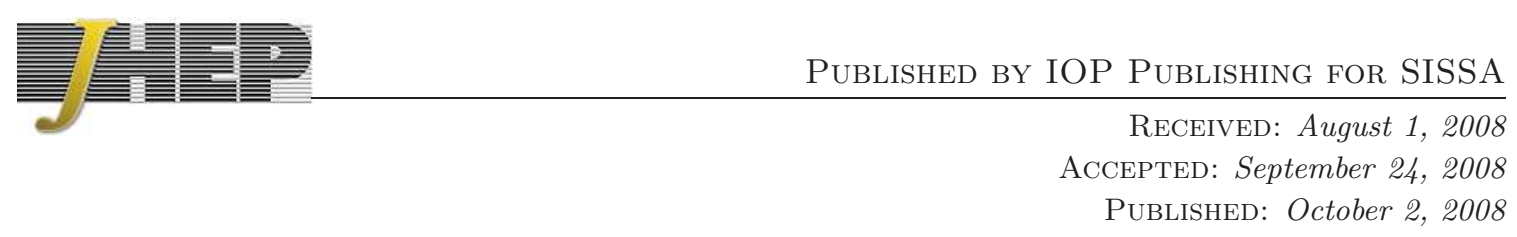

\title{
Sneutrino NLSP scenarios in the NUHM with gravitino dark matter
}

\section{John Ellis}

TH Division, PH Department, CERN,

CH-1211 Geneva 23, Switzerland

E-mail: john.ellis@cern.ch

\section{Keith A. Olive}

William I. Fine Theoretical Physics Institute, University of Minnesota,

116 Church Street S.E., Minneapolis, MN 55455, U.S.A.

E-mail: Olive@physics.umn.edu

\section{Yudi Santoso}

Institute for Particle Physics Phenomenology,

Department of Physics, University of Durham,

South Rd., Durham DH1 3LE, U.K.

E-mail: yudi.santoso@durham.ac.uk

ABSTRACT: We analyze scenarios in which some flavour of sneutrino is the next-to-lightest supersymmetric particle (NLSP), assuming that the gravitino is the lightest supersymmetric particle (LSP) and provides the cold dark matter. Such scenarios do not arise in the constrained supersymmetric extension of the Standard Model (CMSSM) with universal gaugino and scalar masses input at the GUT scale. However, models with non-universal Higgs masses (NUHM) do allow scenarios with a sneutrino NLSP, which are quite generic. We illustrate how such scenarios may arise, analyze the possible metastable sneutrino lifetime, and explore the theoretical, phenomenological, experimental and cosmological constraints on such scenarios. We also discuss the collider signatures of such scenarios, how they may be distinguished from neutralino LSP scenarios, and how different flavours of sneutrino NLSP may be distinguished.

KeYwords: Supersymmetry Phenomenology, Cosmology of Theories beyond the SM. 


\section{Contents}

1. Introduction 且

2. Sneutrino NLSP properties 2

2.1 Sneutrino mass 3

2.2 Sneutrino lifetime

3. Cosmological sneutrino density 5

4. NUHM parameter space 8

5. Cosmological constraints on a sneutrino NLSP 13

6. Signatures of metastable sneutrinos at colliders 16

7. Summary 18

А. Sneutrino three-body decay 19

\section{Introduction}

In the framework of supersymmetry with conserved $R$ parity, the lightest supersymmetric particle (LSP) can have neither electromagnetic nor strong interactions: otherwise it would have bound to conventional matter and been detected in searches for anomalous heavy nuclei 1]. Within the minimal supersymmetric extension of the Standard Model (MSSM), weakly-interacting candidates for the LSP are the lightest sneutrino $\tilde{\nu}$, the lightest neutralino $\chi$, and the gravitino $\tilde{G}$. The (left-'handed') sneutrino LSP hypothesis is excluded by a combination of neutrino counting at LEP and direct dark matter searches [2]. Accordingly, general attention is focused on the neutralino (NDM) [3] and gravitino dark matter (GDM) 4, 5] possibilities, and in this paper we assume the latter.

The next question is the possible nature of the next-to-lightest supersymmetric particle (NLSP) in a GDM model. Two natural possibilities are the other candidates for the LSP, namely the sneutrino and the neutralino, but the NLSP could equally well be charged and even coloured. Indeed, the lighter stau slepton is a natural candidate for the NLSP [6] within the constrained MSSM (CMSSM) with gravity-mediated supersymmetry breaking, in which the soft supersymmetry-breaking scalar masses $m_{0}$, trilinear parameters $A_{0}$ and gaugino masses $m_{1 / 2}$ are each assumed to be universal at a GUT input scale [7, 8]. The lighter stau is also a natural possibility within minimal supergravity (mSUGRA), in which the gravitino mass is fixed: $m_{\tilde{G}}=m_{0}$, and there is an additional relation between trilinear 
and bilinear soft supersymmetry-breaking parameters [9]. Another possibility for the NLSP within a scenario with non-universal Higgs masses (NUHM) 10-14 is the lighter stop [15].

In this paper we study the other possibility for the NLSP within the NUHM with gravity-mediated supersymmetry breaking, namely the lightest sneutrino, assuming that the gravitino provides the cold dark matter. ${ }^{1}$ Like other NLSP candidates in gravitymediated scenarios, the sneutrino NLSP within the NUHM is expected to be very longlived. The dominant decays of the other NUHM NLSP candidates produce particles with copious interactions such as charged particles and photons, which are subject to strong cosmological limits [17]. These limits are strong enough to exclude effectively all of the parameter space where the lightest neutralino is the NLSP [18]. ${ }^{2}$ However, the dominant decay mode of the sneutrino is $\tilde{\nu} \rightarrow \tilde{G} \nu$, and the cosmological limits on neutrino injection are much weaker than those on the injection of photons and charged particles [19, 20]. Therefore the cosmological limits on NUHM $\tilde{\nu}$ NLSP scenarios are relatively weak, leaving considerable scope for a sneutrino NLSP. On the other hand, the sneutrino must appear at the end of the decay chain of every MSSM sparticle produced at a collider, and the particles produced in supersymmetric decay cascades provide distinctive experimental signatures for a sneutrino NLSP [21]. In particular, the charged leptons produced in association with a $\tilde{\nu}$ NLSP provide tools for diagnosing its flavour.

The structure of this paper is as follows. In section 2 we discuss sneutrino properties within the NUHM, including its mass and lifetime. In section 3 we discuss the relic abundance of sneutrinos after freeze-out from a primordial plasma in thermal equilibrium. In section 4 we analyze the NUHM parameter space and identify regions where the NLSP may be either the $\tilde{\nu}_{e, \mu}$ or the $\tilde{\nu}_{\tau}$. In section 5 we discuss the cosmological constraints on $\tilde{\nu}$ NLSP scenarios, and show that they are not severe. In section 6 we discuss some signatures of metastable sneutrinos in different NUHM scenarios, in particular those with different lepton flavours accompanying the $\tilde{\nu}$ NLSP. Our conclusions are summarized in section 7 . Calculations of the three-body decay $\tilde{\nu} \rightarrow \tilde{G} \nu \gamma$ are described in an appendix.

\section{Sneutrino NLSP properties}

We assume that the GUT scale is the effective input scale at which the soft masses are specified, presumably via some gravity-mediated mechanism, and make the NUHM assumptions that the gaugino masses are universal, as are the squark and slepton masses, whereas the soft supersymmetry-breaking contributions to the Higgs scalar masses are non-universal. We then calculate the physical supersymmetric mass parameters at a low energy scale from the running given by the renormalization-group equations (RGEs). We assume that the right-handed neutrino supermultiplets, being singlets, get very large Majorana masses, and therefore decouple from the low-energy effective theory. ${ }^{3}$ The sneutrino NLSP discussed

\footnotetext{
${ }^{1}$ The possibility of a sneutrino NLSP has also been studied within gaugino-mediated models of supersymmetry breaking [16].

${ }^{2}$ If the gravitino mass is much lighter than the neutralino mass, these limits might still be satisfied.

${ }^{3}$ However, if the decoupling energy scale is significantly below the GUT scale, the low-energy spectrum may be affected 22 through the RGEs. This effect is neglected here.
} 
in this paper is essentially the scalar partner of some left-handed neutrino. The flavour of the $\tilde{\nu}$ NLSP is, however, model-dependent, as we discuss below.

\subsection{Sneutrino mass}

In order to calculate the sneutrino mass, we first look at the RGEs of the slepton sec$\operatorname{tor}^{4}\left[23::^{5}\right.$

$$
\begin{aligned}
\frac{d m_{\widetilde{L}_{L}}^{2}}{d t} & =\frac{1}{8 \pi^{2}}\left(-3 g_{2}^{2} M_{2}^{2}-g_{1}^{2} M_{1}^{2}-2 S\right) \\
\frac{d m_{\widetilde{e}_{R}}^{2}}{d t} & =\frac{1}{8 \pi^{2}}\left(-4 g_{1}^{2} M_{1}^{2}+4 S\right) \\
\frac{d m_{\widetilde{L}_{3 L}}^{2}}{d t} & =\frac{1}{8 \pi^{2}}\left(-3 g_{2}^{2} M_{2}^{2}-g_{1}^{2} M_{1}^{2}+h_{\tau}^{2}\left(m_{\widetilde{L}_{3 L}}^{2}+m_{\widetilde{\tau}_{R}}^{2}+m_{1}^{2}+A_{\tau}^{2}\right)-2 S\right), \\
\frac{d m_{\widetilde{\tau}_{R}}^{2}}{d t} & =\frac{1}{8 \pi^{2}}\left(-4 g_{1}^{2} M_{1}^{2}+2 h_{\tau}^{2}\left(m_{\widetilde{L}_{3 L}}^{2}+m_{\widetilde{\tau}_{R}}^{2}+m_{1}^{2}+A_{\tau}^{2}\right)+4 S\right),
\end{aligned}
$$

where

$$
\begin{aligned}
S \equiv \frac{g_{1}^{2}}{4}\left(m_{2}^{2}-m_{1}^{2}+2\left(m_{\widetilde{Q}_{L}}^{2}-m_{\widetilde{L}_{L}}^{2}-2 m_{\widetilde{u}_{R}}^{2}+m_{\widetilde{d}_{R}}^{2}+m_{\widetilde{e}_{R}}^{2}\right)\right. \\
\left.+\left(m_{\widetilde{Q}_{3 L}}^{2}-m_{\widetilde{L}_{3 L}}^{2}-2 m_{\widetilde{t}_{R}}^{2}+m_{\widetilde{b}_{R}}^{2}+m_{\widetilde{\tau}_{R}}^{2}\right)\right) .
\end{aligned}
$$

Here $m_{1,2}$ are the soft masses for the Higgs doublets $H_{1,2} \cdot{ }^{6}$ The $S$ term vanishes and does not contribute in models with universal soft masses such as the CMSSM. However, for non-universal models, $S$ can be far from zero and its contribution to the RGEs can be significant. When $S=0$, and assuming universal soft breaking masses for the $L$ and $R$ sleptons at the GUT scale, the $R$ slepton is lighter than the $L$ slepton at the weak scale. However, since $S$ contributes in opposite ways for $L$ and $R$ sleptons, if $S$ is large and negative, $\widetilde{L}_{L}$ could be lighter than $\widetilde{L}_{R}$. Furthermore, there are additional $D$-terms,

$$
\begin{aligned}
& m_{\tilde{e}_{L}}^{2}=m_{\widetilde{L}_{L}}^{2}-\cos (2 \beta) m_{Z}^{2}\left(\frac{1}{2}-\sin ^{2} \theta_{W}\right), \\
& m_{\tilde{\nu}_{L}}^{2}=m_{\widetilde{L}_{L}}^{2}+\cos (2 \beta) \frac{1}{2} m_{Z}^{2}
\end{aligned}
$$

that split the sneutrino and charged-slepton masses. Since $\cos (2 \beta)<0$ for $\tan \beta>1$, the sneutrino is lighter than its charged-slepton partner.

Comparing the first two generations and the third generation, we see that the tau sneutrino could be lighter than the electron and muon sneutrinos because of the Yukawa terms in the RGEs. However, this is not always the case, due to the fact that $m_{1}^{2}$ could be negative and large, and we display examples later where the $\tilde{\nu}$ NLSP has electron or muon flavour. The lighter stau mass is also suppressed by off-diagonal terms in the mass

\footnotetext{
${ }^{4}$ Although we write the one-loop RGEs for simplicity, our calculations include two-loop contributions.

${ }^{5}$ Note that our convention for $g_{1}^{2}$ is different from [23] by a factor of $5 / 3$. Also note that, in some other papers, e.g. 24, 25], the $S$ term below is assumed to be zero which is valid only for universal case. See also e.g. 26, 27.

${ }^{6}$ Our convention is such that $H_{1,2} \equiv H_{d, u}$, and $\tan \beta \equiv v_{2} / v_{1}$.
} 
matrix. Thus, depending on the model parameters, either the tau sneutrino or the lighter stau might be lighter.

Therefore, a sneutrino could be the NLSP if $S$ is large and negative. ${ }^{7}$ We see from (2.2) that $S$ is negative when $m_{2}^{2}-m_{1}^{2}<0,{ }^{8}$ which is not possible in the CMSSM, for which $S=0$ by assumption. We now study how this may occur in the NUHM model, using the freedom that the Higgs soft supersymmetry-breaking masses at the GUT scale are not necessarily equal to $m_{0}$, the universal scalar mass for sleptons and squarks.

The electroweak symmetry breaking conditions may be written in the form:

$$
m_{A}^{2}(Q)=m_{1}^{2}(Q)+m_{2}^{2}(Q)+2 \mu^{2}(Q)+\Delta_{A}(Q)
$$

and

$$
\mu^{2}=\frac{m_{1}^{2}-m_{2}^{2} \tan ^{2} \beta+\frac{1}{2} m_{Z}^{2}\left(1-\tan ^{2} \beta\right)+\Delta_{\mu}^{(1)}}{\tan ^{2} \beta-1+\Delta_{\mu}^{(2)}},
$$

where $\Delta_{A}$ and $\Delta_{\mu}^{(1,2)}$ are loop corrections [24, 25, 28] and $m_{1,2} \equiv m_{1,2}\left(m_{Z}\right)$. The values of the NUHM parameters at $Q$ are related to their values at $m_{Z}$ through the known radiative corrections [24, 29, 23] $c_{1}, c_{2}$ and $c_{\mu}$ :

$$
\begin{aligned}
m_{1}^{2}(Q) & =m_{1}^{2}+c_{1}, \\
m_{2}^{2}(Q) & =m_{2}^{2}+c_{2}, \\
\mu^{2}(Q) & =\mu^{2}+c_{\mu} .
\end{aligned}
$$

Solving for $m_{1}^{2}$ and $m_{2}^{2}$, one has

$$
\begin{aligned}
m_{1}^{2}\left(1+\tan ^{2} \beta\right)= & m_{A}^{2}(Q) \tan ^{2} \beta-\mu^{2}\left(\tan ^{2} \beta+1-\Delta_{\mu}^{(2)}\right)-\left(c_{1}+c_{2}+2 c_{\mu}\right) \tan ^{2} \beta \\
& -\Delta_{A}(Q) \tan ^{2} \beta-\frac{1}{2} m_{Z}^{2}\left(1-\tan ^{2} \beta\right)-\Delta_{\mu}^{(1)}
\end{aligned}
$$

and

$$
\begin{aligned}
m_{2}^{2}\left(1+\tan ^{2} \beta\right)= & m_{A}^{2}(Q)-\mu^{2}\left(\tan ^{2} \beta+1+\Delta_{\mu}^{(2)}\right)-\left(c_{1}+c_{2}+2 c_{\mu}\right) \\
& -\Delta_{A}(Q)+\frac{1}{2} m_{Z}^{2}\left(1-\tan ^{2} \beta\right)+\Delta_{\mu}^{(1)}
\end{aligned}
$$

The correction $\Delta_{\mu}^{(2)}$ is positive and generally of $O(0.1)$. From here we can see that there are two possible ways to get negative $S$ via negative $m_{2}^{2}-m_{1}^{2}$ : the first is by using very large $\mu^{2}$, and the second is by using very large $m_{A}^{2}$. If $m_{A}^{2}$ is relatively small while $\mu^{2}$ is very large, the Higgs masses-squared $m_{H_{1,2}}^{2} \equiv m_{1,2}^{2}+\mu^{2}$ may be negative at the GUT scale, which could lead to a vacuum stability problem [30].

A weak-scale scalar mass-squared with $m^{2}\left(M_{\mathrm{GUT}}\right)<0$ generally produces a vev of order the weak scale that disappears as the RGEs are run down to the weak scale. Such

\footnotetext{
${ }^{7}$ One could also obtain a light sneutrino within a supersymmetric SU(5) GUT with different soft masses for the $\mathbf{1 0}$ and $\overline{\mathbf{5}}$ multiplets $[20$. Another alternative is within a gaugino-mediated supersymmetry breaking model, in which the Higgs masses are again different from the other sfermion masses 16 .

${ }^{8}$ Assuming that these are the dominant terms in $S$, which is the case for the NUHM that we consider here.
} 
negative masses-squared are not dangerous. It may happen, however, that an instability occurs along some $F$ - and $D$ - flat direction. In this case, a negative mass-squared may be large and still present at a renormalization scale, $Q \sim v$ 31. Models in which the Universe becomes trapped in such non-Standard Model vacua are clearly excluded. ${ }^{9}$ This possibility has been studied in the NUHM along the $H_{1}-H_{2}$ and $H_{2}-L$ flat directions of the MSSM [33]. We delineate below regions in the parameter plane where these vacua may be problematic.

\subsection{Sneutrino lifetime}

In the GDM scenario used here, the sneutrino NLSP would eventually decay into the gravitino, and the dominant decay channel is the two-body decay

$$
\widetilde{\nu} \rightarrow \widetilde{G}+\nu
$$

with the decay rate

$$
\Gamma_{2 \mathrm{~b}}=\frac{1}{48 \pi} \frac{m_{\tilde{\nu}}^{5}}{M_{\mathrm{Pl}}^{2} m_{\widetilde{G}}^{2}}\left(1-\frac{m_{\widetilde{G}}^{2}}{m_{\tilde{\nu}}^{2}}\right)^{4},
$$

where $m_{\widetilde{G}}$ is the gravitino mass and $M_{\mathrm{Pl}}$ is the Planck mass: $M_{\mathrm{Pl}}=1 / \sqrt{8 \pi G_{N}} \simeq$ $2.4 \times 10^{18} \mathrm{GeV}$.

We plot in figure 1 the sneutrino lifetime, $\tau_{\tilde{\nu}} \simeq 1 / \Gamma_{2 \mathrm{~b}}$, as a function of the gravitino mass for $m_{\tilde{\nu}}=10,100,500$, and $1000 \mathrm{GeV}$ respectively. Note that we plot the lifetime only for $\Delta m \equiv m_{\tilde{\nu}}-m_{\widetilde{G}} \geq 1 \mathrm{GeV}$. Clearly, a smaller mass gap would yield an even longer lifetime. We see that the sneutrino lifetime could be less than 1 second only when $m_{\tilde{\nu}}$ is large, or the gravitino mass is (much) less than $1 \mathrm{GeV}$. On the other hand, if the mass gap is small, the sneutrino lifetime can be very long, potentially even longer than the age of the Universe, which is $\mathcal{O}\left(10^{17}\right)$ s. However, there are cosmological and astrophysical constraints on the possibility of a sneutrino with lifetime longer than the age of the Universe at recombination that we discuss in more detail later.

\section{Cosmological sneutrino density}

Assuming thermal equilibrium in the early Universe, one can calculate the sneutrino relic density after decoupling but before its decay into the gravitino. This is done by the usual use of the Boltzmann equation and calculation of the sneutrino annihilation and coannihilation cross sections. The calculations are identical to those required to calculate the relic sneutrino density if it is the LSP.

The possible sneutrino-pair annihilation two-body final states [12] are listed in table 1. If the soft masses for the sfermions are universal as assumed here, the electron sneutrino is always degenerate with the muon sneutrino, and the tau-sneutrino mass might be nearby. In the NUHM case that we consider here, there could also be other sparticles that are almost degenerate with the sneutrinos, such as the lightest neutralino and chargino, and charged sleptons. We list the corresponding coannihilation processes and their possible final states in tables $2-5$. 


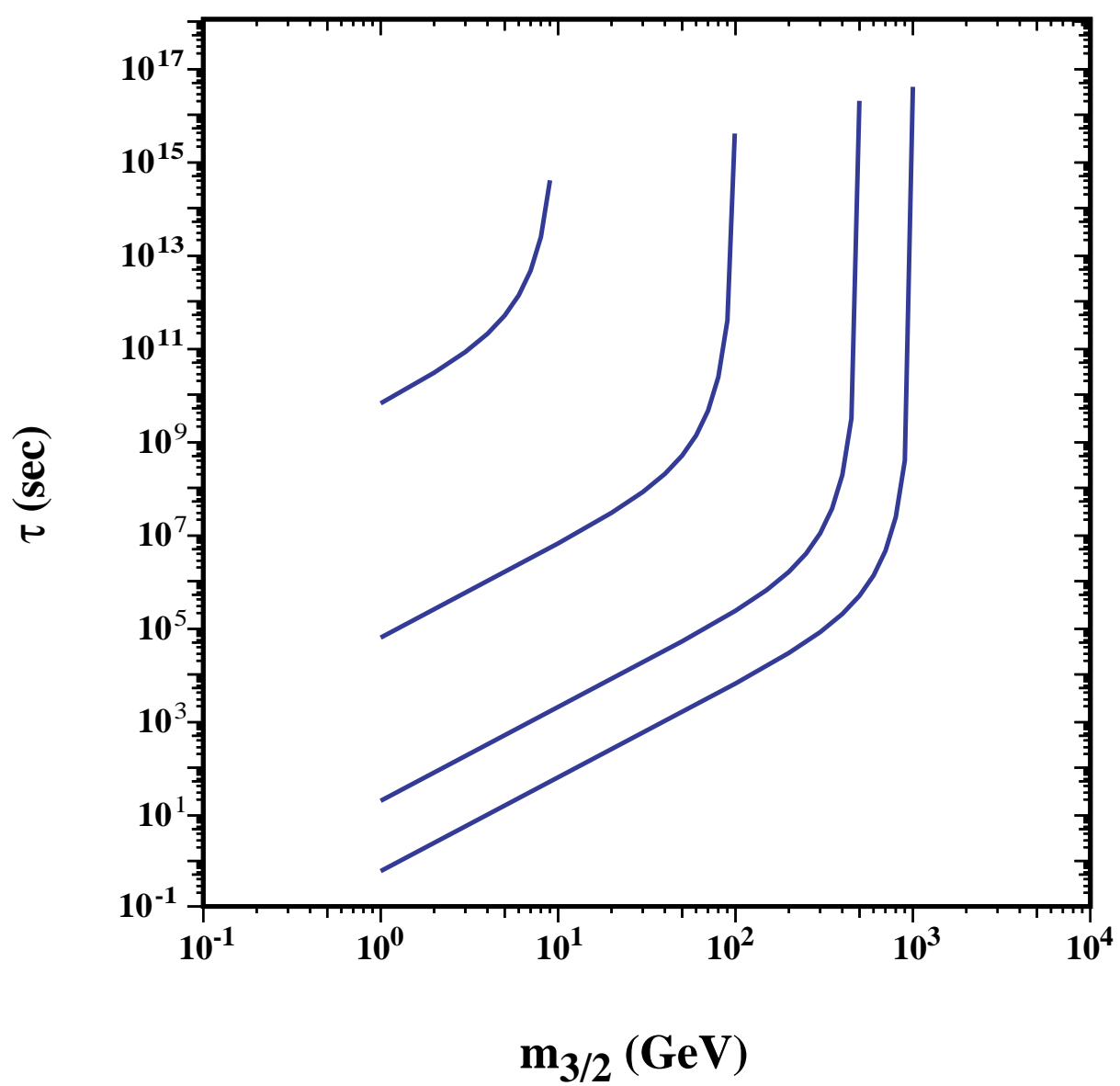

Figure 1: The sneutrino NLSP lifetime as a function of $m_{\widetilde{G}}$ for $m_{\tilde{\nu}}=10,100,500$ and $1000 \mathrm{GeV}$ (top to bottom).

\begin{tabular}{|c|l|}
\hline Initial & Final States \\
State & \\
\hline & \\
$\tilde{\nu}_{i} \tilde{\nu}_{i}^{*}$ & $f \bar{f}, W^{+} W^{-}, Z Z, h Z, h A, H Z, H A, h h, h H, H H, A A, A Z, H^{+} H^{-}, W^{+} H^{-}, H^{+} W^{-}$ \\
$\tilde{\nu}_{i} \tilde{\nu}_{i}$ & $\nu_{i} \nu_{i}$
\end{tabular}

Table 1: Sneutrino pair annihilation final states. There are also some other modes listed in table 2.

In our scenario, the sneutrino NLSP eventually decays into the gravitino before the current epoch. Consequently, the gravitino relic density is related to the sneutrino density before its decay by

$$
\Omega_{\tilde{G}} h^{2}=\frac{m_{\widetilde{G}}}{m_{\tilde{\nu}}} \Omega_{\tilde{\nu}} h^{2}+\Omega_{\tilde{G}}^{\mathrm{T}} h^{2},
$$

where $\Omega_{\tilde{G}}^{T}$ is the contribution to the gravitino density from thermal production after re-

\footnotetext{
${ }^{9}$ Whether this occurs or not depends on the specific cosmological history during inflation 32, 33.
} 


\begin{tabular}{|c|l|}
\hline Initial State & Final States \\
\hline & \\
$\tilde{\nu}_{i} \tilde{\nu}_{j}^{*}$ & $\nu_{i} \bar{\nu}_{j}, \ell_{i} \bar{\ell}_{j}$ \\
$\tilde{\nu}_{i} \tilde{\nu}_{j}$ & $\nu_{i} \nu_{j}$ \\
\hline
\end{tabular}

Table 2: Sneutrino (co)annihilation with other sneutrino flavours. For these modes we can either have $i=j$ (i.e. pair annihilation) or $i \neq j$.

\begin{tabular}{|c|l|}
\hline Initial State & Final States \\
\hline & \\
$\tilde{\ell}_{i} \tilde{\nu}_{i}^{*}$ & $f \bar{f}^{\prime}, h H^{-}, H H^{-}, A H^{-}, h W^{-}, H W^{-}, Z W^{-}, \gamma W^{-}, W^{-} A$ \\
$\tilde{\ell}_{i} \tilde{\nu}_{j}^{*}$ & $\ell_{i} \bar{\nu}_{j}$ \\
$\tilde{\ell}_{i} \tilde{\nu}_{j}$ & $\ell_{i} \nu_{j}, \nu_{i} \ell_{j}$ \\
\hline
\end{tabular}

Table 3: Sneutrino coannihilation with charged sleptons. In the first line above, the coannihilation is between a sneutrino and a charged slepton of the same generation, whereas in the second and third lines they are not necessarily from the same generation.

\begin{tabular}{|c|l|}
\hline Initial State & Final States \\
\hline$\chi \tilde{\nu}$ & $\nu Z, \nu h, \nu H, \nu A, \ell^{-} W^{+}, \ell^{-} H^{+}$ \\
\hline
\end{tabular}

Table 4: Sneutrino coannihilation with neutralino. Similar modes are available for $\widetilde{\nu}^{*}$.

\begin{tabular}{|c|l|}
\hline Initial State & Final States \\
\hline$\chi^{-} \tilde{\nu}$ & $\ell \gamma, \ell Z, \ell h, \ell H, \ell A, \nu W^{-}, \nu H^{-}$ \\
$\chi^{+} \tilde{\nu}$ & $\nu W^{+}, \nu H^{+}$ \\
\hline
\end{tabular}

Table 5: Sneutrino coannihilation with chargino. Similarly for $\widetilde{\nu}^{*}$.

heating, which is sensitive to the unknown reheating temperature $T_{R}$. We do not discuss this contribution here. The only constraint we impose is that the contribution to the gravitino relic density arising from sneutrino decay does not exceed the value suggested by WMAP [34] and other observations:

$$
\Omega_{\mathrm{DM}} h^{2}=0.1099 \pm 0.0062 .
$$

Hence, we require that the first term on the right-hand side of (3.1) should not exceed $\sim 0.1223$ (the $2-\sigma$ upper limit). Because of the scaling by the mass ratio $m_{\widetilde{G}} / m_{\tilde{\nu}}$, even a large sneutrino density after decoupling could still be compatible with the dark matter constraint if $m_{\widetilde{G}} \ll m_{\tilde{\nu}}$.

In this case, we must check whether gravitinos are non-relativistic at the time structure formation begins, roughly at $t_{s} \simeq 5 \times 10^{11} \mathrm{~s}$, in which case they act in the same way as 
conventional cold dark matter. If $m_{3 / 2} \ll m_{\tilde{\nu}}, E_{3 / 2} / m_{3 / 2} \simeq m_{\tilde{\nu}} / 2 m_{3 / 2}$ and $E_{3 / 2}$ scales subsequently as $\left(\tau_{\tilde{\nu}} / t\right)^{1 / 2}$, where $\tau_{\tilde{\nu}}$ is the sneutrino lifetime. We can use figure 1 to estimate whether or not gravitinos will behave as cold dark matter. For example, at the left-most point of the lowest curve, gravitinos are produced relativistically with $E_{3 / 2} / m_{3 / 2} \sim 1000$, but the decay occurs so early that they become non-relativistic well before structure formation begins. The same is also true for the two middle curves in figure 1. Only for the left part of the topmost curve (when $m_{\tilde{\nu}}=10 \mathrm{GeV}$ ) is there a potential problem. However, in this case the sneutrino relic density is generally already small in the model considered here.

\section{NUHM parameter space}

In the CMSSM, the values of $m_{A}$ and $\mu$ are determined by the electroweak vacuum conditions for any given input values of $m_{1 / 2}, m_{0}, A_{0}$ and $\tan \beta$. However, the constraints on $m_{A}$ and $\mu$ are relaxed in the NUHM, with the values of these parameters being related to the degrees of non-universality assumed for the Higgs soft masses $m_{1}^{2}$ and $m_{2}^{2}$. A general discussion of the parameter space of the NUHM was given in [11-14, which we use here as a starting point for our discussion. Regions of the NUHM parameter space in which a sneutrino is the lightest spartner of any Standard Model particle were identified in [12, see for example the dark blue shaded regions in figures 2, 3, 4, 6, 8 and 9 of that paper. There, it was assumed that the LSP is the lightest neutralino $\chi$, with the gravitino assumed to be heavy, so that these light-sneutrino regions were disallowed. However, in this paper we assume that the gravitino is the LSP, so the viability of these light-sneutrino regions must be re-evaluated. We focus our discussion here on $\left(\mu, m_{A}\right)$ planes of the types shown in figures 4 and 6 of [12], where the light-sneutrino region moves to lower $|\mu|$ as $m_{A}$ increases.

In general, keeping some sneutrino species light favours small values of $m_{1 / 2}$ and $m_{0}$. However, there is an important lower limit on $m_{1 / 2}$, in particular, due to the LEP lower bound on the mass of the lightest MSSM Higgs boson. A sneutrino NLSP region may be found by choosing a moderate value of $m_{1 / 2}=500 \mathrm{GeV}$ while keeping $m_{0}$ relatively small, e.g., $m_{0}=100 \mathrm{GeV}$. The resulting masses of the sparticles are shown in figure 2 assuming $\tan \beta=10$ and $A_{0}=0$, for various values of $m_{A}=200,1000,1500$ and $2000 \mathrm{GeV}$, in panels (a), (b), (c), and (d), respectively. ${ }^{10}$

As noted earlier, in regions of the NUHM parameter plane (particularly when $\mu$ and $m_{A}$ are large), the masses-squared of the Higgs and left-sleptons have a tendency to run down to negative values at the GUT scale. This allows for the possibility that large scale vevs be excited along the $H_{1}-H_{2}$ or $H_{2}-L$ flat directions. One expects that these flat directions are lifted by some effective operator at or above the GUT scale. The vev along the flat direction is sensitive to the fundamental scale associated with this operator and clearly grows as that scale is increased above the GUT scale. The reliability of this high-scale vacuum depends also on the one-loop corrections to the scalar potential. This sensitivity can be characterized by the ratio of the tree-level vev to the renormalization scale, $Q_{0}$, at which the vev disappears (i.e., the masses-squared go positive). Here we

\footnotetext{
${ }^{10}$ We use $m_{t}=172.6 \mathrm{GeV}$ for our analysis 35.
} 

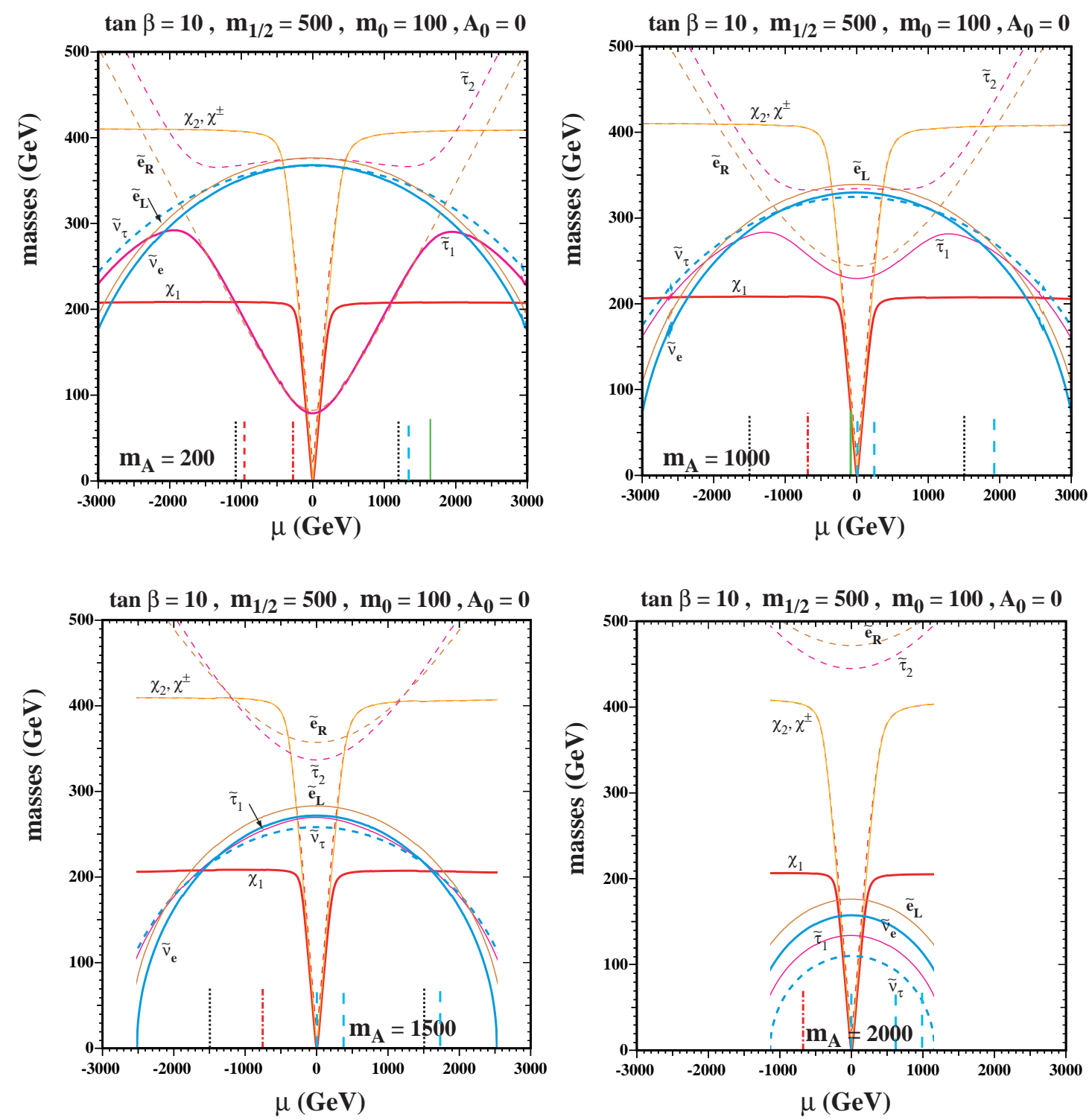

Figure 2: Sparticle masses as functions of $\mu$ for $\tan \beta=10, m_{1 / 2}=500 \mathrm{GeV}, m_{0}=100 \mathrm{GeV}$, $A_{0}=0, m_{t}=172.6 \mathrm{GeV}, m_{b}\left(m_{b}\right)^{\overline{\mathrm{MS}}}=4.25 \mathrm{GeV}$, and $m_{A}=$ (a) $200 \mathrm{GeV}$, (b) $1000 \mathrm{GeV}$, (c) $1500 \mathrm{GeV}$ and (d) $2000 \mathrm{GeV}$, respectively. In panels (c) and (d), the sparticle lines are truncated at larger $|\mu|$ where some sneutrino becomes tachyonic. Constraints are represented by vertical lines: black dotted for the GUT constraint (larger $|\mu|$ is excluded); red dot-dashed shows the Higgs mass contour at $m_{h}=114.4 \mathrm{GeV}$, while the constraint using the LEP likelihood function convolved with theoretical uncertainties in the Higgs mass (computed here using FeynHiggs [36]) is shown by the red dashed line; the $(g-2)_{\mu}$ constraint (described in the text) is shown by the light blue long dashed lines; and solid green for the $b \rightarrow s \gamma$ constraint (smaller $\mu$ is excluded).

adopt the most conservative set of assumptions, namely that the flat directions are lifted at the GUT scale and that the vev must be of order $Q_{0}(\epsilon=1$ in the notation of [33). This preserves the largest volume of the NUHM parameter space. Of course the GUT constraint 
itself is cosmology-dependent, and may not be important if the Universe starts out in the weak scale vacuum after inflation. For more details on this constraint see [33].

In the case of small $m_{A}=200 \mathrm{GeV}$, shown in panel (a) of figure 2, we see that the GUT stability constraint (represented by a couple of vertical black dotted lines) allows only small $|\mu| \lesssim 1.1 \mathrm{TeV}$, far from the sneutrino NLSP region which appears when $|\mu| \gtrsim 2800 \mathrm{GeV}$. We also see that the $b \rightarrow s \gamma$ constraint (solid green line) allows only $\mu \gtrsim 1600 \mathrm{GeV}$ and the Higgs mass constraint (dashed red) allows only $\mu \gtrsim-1000 \mathrm{GeV}$ for this small value of $m_{A}$. Note that a Higgs mass of $114.4 \mathrm{GeV}$ is found around $\mu \simeq-300 \mathrm{GeV}$ (dot-dashed vertical line) for this value of $m_{A}$, but current theoretical and experimental uncertainties only provide for the weaker bound shown by the dashed line. The anomalous magnetic moment of the muon, $(g-2)_{\mu}$, is reconciled with experiment at the $95 \%$ CL for $\mu \gtrsim 1300 \mathrm{GeV}$, as shown by the light blue long-dashed line. ${ }^{11}$ Therefore, we do not have an allowed region in panel (a), assuming the GUT constraint holds. For larger $m_{A}$, these constraints become more relaxed, and an allowed sneutrino NLSP region emerges.

For $m_{A}=1000 \mathrm{GeV}$, shown in panel (b) of figure 2, the GUT stability constraint allows $|\mu| \lesssim 1.5 \mathrm{TeV}$, the $b \rightarrow s \gamma$ constraint allows $\mu \gtrsim 0$, and the Higgs constraint is essentially unimportant, as a Higgs mass greater than $114.4 \mathrm{GeV}$ occurs at $\mu \gtrsim-700 \mathrm{GeV}$. The $(g-2)_{\mu}$ constraint is satisfied in two regions: $0<\mu<250 \mathrm{GeV}$ and $\mu \gtrsim 1900 \mathrm{GeV}$. While the GUT constraint is relaxed, the sneutrino LSP region still requires $2.4 \mathrm{TeV} \lesssim|\mu|$, where we have degenerate $\tilde{\nu}_{e, \mu}$ NLSPs. We note that around $\mu=2.5 \mathrm{TeV}$ several other sparticles are only slightly heavier than the $\tilde{\nu}_{e, \mu}$, including the lightest neutralino $\chi$, the $\tilde{e}_{L}$ and $\tilde{\mu}_{L}$, the lighter $\tilde{\tau}$ and the $\tilde{\nu}_{\tau}$. Thus, all these sparticles must be included in coannihilation calculations of the $\tilde{\nu}_{e, \mu}$ abundance. For larger $\mu$, only $\tilde{e}_{L}$ and $\tilde{\mu}_{L}$ masses stay close to the NLSP mass, while the others get larger mass gaps.

When $m_{A}$ is increased to $1500 \mathrm{GeV}$, as shown in panel (c) of figure 2, the GUT constraint remains at $|\mu| \lesssim 1.5 \mathrm{TeV}$, very close to the region when the sneutrino is the NLSP, which extends from $\mu \simeq 1.6 \mathrm{TeV}$ to $\simeq 2.5 \mathrm{TeV}$. The lightest sparticles are again the $\tilde{\nu}_{e, \mu}$, with the $\chi, \tilde{e}_{L}, \tilde{\mu}_{L}, \tilde{\tau}_{1}$ and $\tilde{\nu}_{\tau}$ again slightly heavier. In this case, a theoretical upper limit on $|\mu|$ arises when the $\tilde{\nu}_{e, \mu}$ become tachyonic. ${ }^{12}$ In this case, the Higgs constraint requires only that $\mu \gtrsim-1300 \mathrm{GeV}$, and $(g-2)_{\mu}$ is satisfied when $0<\mu \lesssim 400 \mathrm{GeV}$ or $\mu \gtrsim 1700 \mathrm{GeV}$. The constraint from $b \rightarrow s \gamma$ is unimportant at this value of $m_{A}$.

Finally, in panel (d) of figure 2 we display the sparticle masses for $m_{A}=2000 \mathrm{GeV}$. In this case, the allowed sneutrino NLSP is the $\tilde{\nu}_{\tau}$, for $200 \mathrm{GeV} \lesssim|\mu| \lesssim 1.1 \mathrm{TeV}$. The $\chi$ becomes the NLSP for smaller $|\mu|$ in the Higgsino region, and upper limits are provided by the LEP lower limits discussed above. For this value of $m_{A}$, the differences in mass between the $\tilde{\nu}_{\tau}$ and the heavier sparticles are relatively large. Neither the Higgs mass nor $b \rightarrow s \gamma$ provide a constraint, while $(g-2)_{\mu}$ requires $0<\mu \lesssim 600 \mathrm{GeV}$ or $\mu \gtrsim 1000 \mathrm{GeV}$ with the later has $m_{\tilde{\nu}}$ less than the LEP limit.

In these plots, we find that sneutrino NLSP has relic density of order $O\left(10^{-3}\right)$ which

\footnotetext{
${ }^{11}$ We assume that the deviation of $(g-2)_{\mu} / 2$ from the standard model is between 10.7 to $44.3 \times 10^{-10}$, the $2 \sigma$ range according to 37 .

${ }^{12}$ Here and in panel (d), we truncate all the other sparticle lines at this boundary of the tachyonic region. Slightly more stringent upper limits on $|\mu|$ come from the lower limits on $m_{\tilde{\nu}}$ provided by LEP [38, 39.
} 
is well below the WMAP limit. This means that most of the gravitino dark matter must be produced by some other sources, e.g., by reheating. We take coannihilation effects into account for the relic density calculation. However, in contrast with the neutralino LSP case, coannihilations do not always reduce the final relic density. Sneutrino coannihilation with the lightest neutralino would indeed generally increase the relic density, while that with charged sleptons might reduce it. In the former (latter) case, the effective sneutrino cross section is averaged with the weaker (stronger) annihilation cross section of neutralinos (charged sleptons). The relatively small relic density of the sneutrino compared to that of the neutralino can be attributed generically to the fact that the sneutrino is a scalar particle, rather than a Majorana fermion. In figure (2(d), for example, since the mass gaps with other sparticles are relatively large, the coannihilation effects are not maximal, but the relic density is still small.

To get a more comprehensive view of the NUHM parameter space, we display in figure 35 some contour plots in selected $\left(\mu, m_{A}\right)$ planes. In panel (a), we choose $\tan \beta=10$, $m_{1 / 2}=500 \mathrm{GeV}, m_{0}=100 \mathrm{GeV}$ and $A_{0}=0$. This panel therefore includes and extends the specific examples shown in figure 2. We plot the regions where the lighter stau, the right selectron, some sneutrino or the lightest neutralino is the NLSP. At large $|\mu|$, the electron-sneutrino is the NSLP and is shown by the regions shaded dark blue. At large $m_{A}$ and smaller $|\mu|$, the NLSP becomes the tau-sneutrino (shaded light blue). Above these regions, the white (blank) area corresponds to an unphysical region where one or more of the sparticles has a negative mass-squared at the weak scale. Below these regions, in most of the area, it is the lightest neutralino which is the NLSP. At lower $m_{A}$ and relatively small $|\mu|$, we see regions where the lighter stau (shaded brick red), or the right selectron (shaded orange) is the NLSP. At even smaller $|\mu|$ the NLSP is a higgsino-like neutralino. The narrow turquoise shaded region is that in which $\Omega_{\mathrm{NLSP}} h^{2}$ is within two $\sigma$ of the WMAP value. (Recall that, with the gravitino as the LSP, this is not the dark matter relic density.) Regions surrounded by the strip (which have higher $\Omega_{\mathrm{NLSP}} h^{2}$ ) might also be permitted if $m_{\widetilde{G}} \ll m_{\mathrm{NLSP}}$, and regions not surrounded by the strip (which have lower $\Omega_{\mathrm{NLSP}} h^{2}$ ) would certainly be permitted by the dark matter constraint. As one can see these regions track very closely the degeneracy lines between the neutralino and one of the four sparticles where the relic density is controlled by coannihilations or the funnel region where $2 m_{\chi} \simeq m_{A}$ (The contour $2 m_{\chi}=m_{A}$ is shown by the thin blue line.).

The Higgs mass contour of $114.4 \mathrm{GeV}$ (red dot-dashed line) excludes $\mu \lesssim-700 \mathrm{GeV}$. However, if one uses a likelihood analysis for the Higgs mass and allows for theoretical uncertainties in the calculation of $m_{h}$, this constraint is relaxed and most of the displayed region is allowed, as shown by red dashed line. The $b \rightarrow s \gamma$ constraint (shaded green) excludes small $m_{A}$ and prefers positive $\mu$. However, this constraint is unimportant in this panel at large $m_{A}$. The GUT constraint is represented by a black dotted line: it excludes large $|\mu|$ and essentially all of the electron-sneutrino NLSP region for this set of parameters. Finally, the light pink shaded region bordered by a black solid line represents the region favored by $(g-2)_{\mu}$. The vertical dashed black lines correspond to the chargino mass contours of $104 \mathrm{GeV}$ and exclude very small values of $|\mu|$.

In panel (b), we display a case with $A_{0}=1000 \mathrm{GeV}$, with the other parameters chosen 

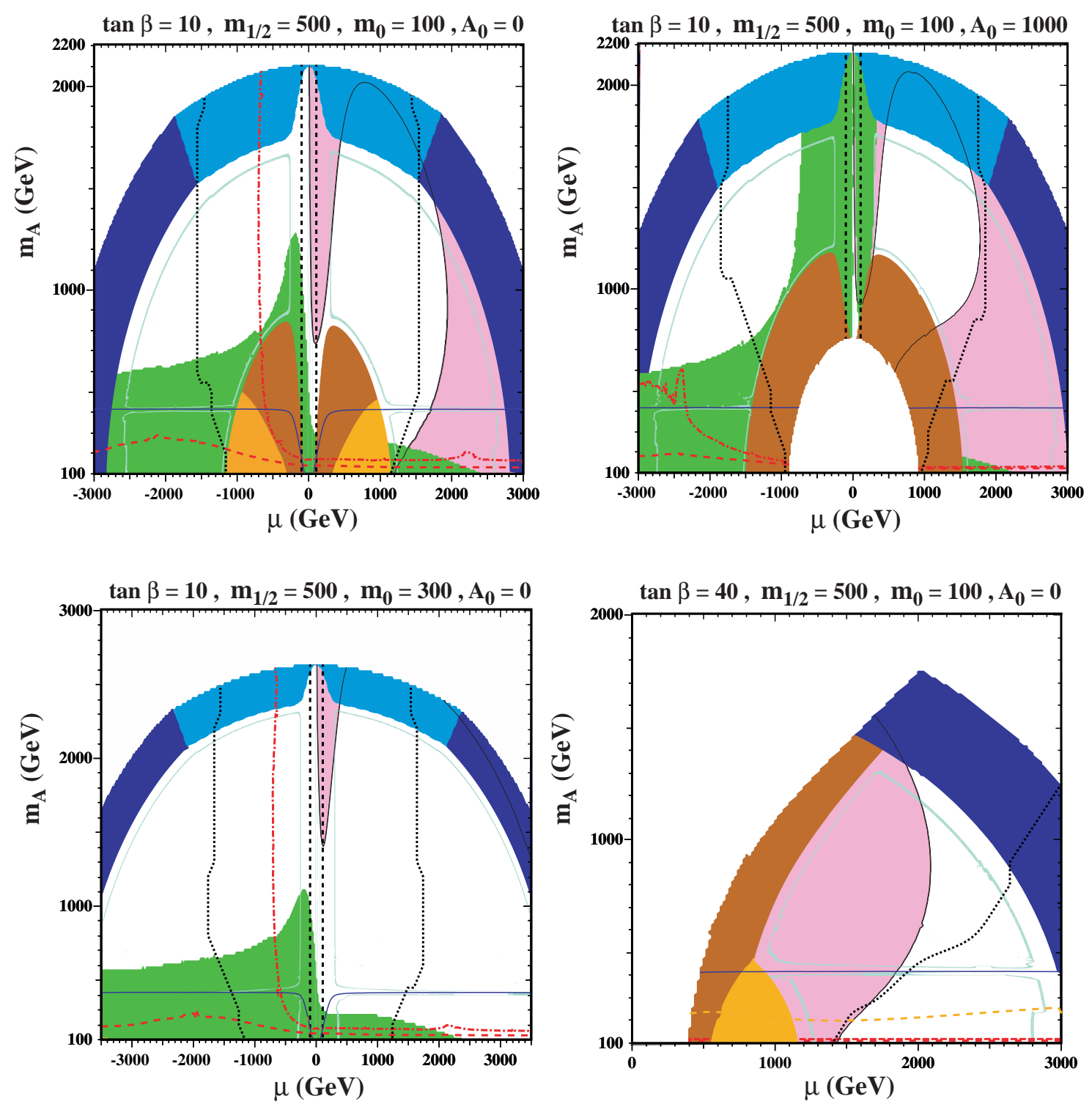

Figure 3: Some $\left(\mu, m_{A}\right)$ planes in the NUHM for (a) $\tan \beta=10, m_{1 / 2}=500 \mathrm{GeV}, m_{0}=100 \mathrm{GeV}$, and $A_{0}=0$; (b) $\tan \beta=10, m_{1 / 2}=500 \mathrm{GeV}, m_{0}=100 \mathrm{GeV}$, and $A_{0}=1000 ;$ (c) $\tan \beta=10$, $m_{1 / 2}=500 \mathrm{GeV}, m_{0}=300 \mathrm{GeV}$, and $A_{0}=0 ;$ (d) $\tan \beta=40, m_{1 / 2}=500 \mathrm{GeV}, m_{0}=100 \mathrm{GeV}$, and $A_{0}=0$. In each case, we used $m_{t}=172.6 \mathrm{GeV}$ and $m_{b}\left(m_{b}\right)^{\overline{\mathrm{MS}}}=4.25 \mathrm{GeV}$. Contours and shading are described in the text.

to be the same as in (a). We see that the stau NLSP region becomes bigger and there is no longer a right-selectron NLSP region. In the white (blank) region interior to the stau NLSP region, the stau has gone tachyonic at the weak scale, which is problematic. We also see that the $b \rightarrow s \gamma$ constraint becomes stronger, especially for negative $\mu$. However, the qualitative features of the sneutrino NLSP band at large $|\mu|$ and/or $m_{A}$ are similar. This feature is also retained in panel (c), where the larger value $m_{0}=300 \mathrm{GeV}$ is chosen, and also in panel 
(d), in which a larger value $\tan \beta=40$ is chosen. In panel (c) (which now extends to higher values of $m_{A}$ ), there is no longer a charged NLSP. In panel (d), we see again a region with a right selectron NLSP. For this value of $\tan \beta$, negative $\mu$ is not allowed by electroweak symmetry breaking condition [12]. The GUT constraint in this case constrains only the lower right corner of the plane shown, and allows part of the electron-sneutrino region. The $B_{s} \rightarrow \mu^{+} \mu^{-}$constraint (orange dashed line) excludes a region with small $m_{A}$ [40].

We conclude that the possibility of a sneutrino NLSP is quite generic in the NUHM, and certainly not much less plausible than the lighter stau. This is in contrast to the CMSSM, where a stau NLSP is a generic feature at large $m_{1 / 2}$ and small $m_{0}$, but there is no possibility of a sneutrino NLSP.

\section{Cosmological constraints on a sneutrino NLSP}

The cosmological impact of a long-lived sneutrino depends on its lifetime. ${ }^{13}$ If the sneutrino decays during or after Big-Bang Nucleosynthesis (BBN), it could alter predictions for the light-element abundances. If the sneutrino decays around or after the time of recombination, it could distort the blackbody spectrum of the CMB. If the sneutrino decays at a very late time, its effect might be seen on the diffuse neutrino and photon spectra. The production of relativistic neutrinos by sneutrino decays could also change the equation of state and therefore the evolution history of the Universe [41].

Although the sneutrino is neutral, and its dominant two-body decay channel produces only a neutrino and the gravitino, which are also neutral, there could still be a significant effect on BBN if the sneutrino decays during or round after the time of BBN [19, 20, 42, 43]. If the mass gap is sufficiently large, the decay of the sneutrino produces high-energy, nonthermal neutrinos. Through scattering processes with the background particles, such as $\nu_{i}+\bar{\nu}_{j, \mathrm{BG}} \rightarrow\left(e^{ \pm}, \mu^{ \pm}, \tau^{ \pm}\right), \nu_{i}+\bar{\nu}_{i, \mathrm{BG}} \rightarrow \pi^{+}+\pi^{-}$and $\nu_{i}+e_{i, \mathrm{BG}}^{ \pm} \rightarrow \pi^{0}+\pi^{ \pm}$, the energetic neutrinos transfer some parts of their energies to charged particles. The final-state particles may then photodissociate or hadrodissociate the elements already produced by standard $\mathrm{BBN}$ processes. In the case of the charged pion, it can alter the neutron-to-proton ratio if it occurs at the beginning of BBN. There can also be energy transfer through elastic scattering with electrons and positrons: $\nu_{i}+e^{ \pm} \rightarrow \nu_{i}+e^{ \pm}$, and the high-energy $e^{ \pm}$might then initiate electromagnetic showers. However, at the epoch of interest (when their energies are $\mathcal{O}(1) \mathrm{MeV}$ ) the electron and positron number densities are already low. Therefore these processes can be neglected.

Subdominant three- and four-body sneutrino decay channels can also be important, even though their branching ratios are relatively small. This is because these decays produce charged and/or strongly-interacting particles directly. These effects had been studied in [19], where it was found that the effects of the three- and four-body decays are negligible if their collective branching ratio is less than about $10^{-6}$. To estimate this branching ratio, we calculate the decay rate for the following process

$$
\widetilde{\nu} \rightarrow \widetilde{G}+\nu+\gamma
$$

\footnotetext{
${ }^{13}$ See [19] and references therein.
} 
which occurs through neutralinos exchange, with the photon produced via the photino content of the neutralinos. This provides an estimate of the total multi-body decay rate that should be accurate to within an order of magnitude. The detailed calculation can be found in the appendix.

To gauge the possible impact of the BBN constraint, we examine the tau sneutrino NLSP region in figure 2(d), where the sneutrino mass varies from the LEP lower limit of about $40 \mathrm{GeV}$ up to about $100 \mathrm{GeV}$. We first consider the case $m_{1 / 2}=500 \mathrm{GeV}$, $m_{0}=100 \mathrm{GeV}, \tan \beta=10, A_{0}=0$ and $m_{A}=2000 \mathrm{GeV}$ shown in the top two panels of figure 4. Panel (a) shows the three-body-decay branching ratio for various values of $m_{\widetilde{G}}=1,10,30 \mathrm{GeV}$, corresponding to $\widetilde{\nu}$ lifetimes $\gtrsim 10^{5} \mathrm{~s}$. We see that the branching ratio is always very small, falling below $10^{-6}$ throughout the range of parameter space considered. This is consistent with the results of [20] which also finds a small hadronic fraction when the sneutrino mass is $\lesssim 100 \mathrm{GeV}$. Thus, according to the analysis of [19], the three-body $\widetilde{\nu}$ decay is too small to affect significantly the successful results of BBN.

Although the hadronic branching ratio is expected to be small, BBN nevertheless sets a limit on the density of sneutrinos at the time of decay. We can see from figures 5 and 8 of [19] that, for a $100 \mathrm{GeV}$ sneutrino, all lifetimes are safe so long as the quantity

$$
Y_{\tilde{\nu}} M_{\tilde{\nu}}=\left(\rho_{c} / s\right) \Omega_{\tilde{\nu}} \simeq\left(3.65 \times 10^{-9} \mathrm{GeV}\right) \times \Omega_{\tilde{\nu}} h^{2}
$$

is less than $\mathcal{O}\left(10^{-11}\right) \mathrm{GeV}$ for $B_{h}=10^{-3}$ and less than $\mathcal{O}\left(10^{-8}\right)$ for $B_{h}=10^{-6}$, where $Y_{\tilde{\nu}}$ is the ratio of the number density of sneutrinos to entropy, $n_{\tilde{\nu}} / s$, and $\rho_{c}$ is the critical density. In panel (b) of figure 4 , we display the sneutrino relic density following freeze-out but prior to decay as $Y_{\tilde{\nu}} M_{\tilde{\nu}}$. We see that $Y_{\tilde{\nu}} M_{\tilde{\nu}}$ is always below about $10^{-11} \mathrm{GeV}$, with a large dip at $m_{\tilde{\nu}} \sim 45 \mathrm{GeV}$ due to the $Z$ resonance in sneutrino-pair annihilation (with a smaller dip at $m_{\tilde{\nu}} \sim 60 \mathrm{GeV}$ due to the $h$ resonance). Thus, the sneutrino density is far below the range where BBN constraints become important for the range of the three-body branching ratio shown in panel (a) of figure 4 .

The lower panels of figure 1 display the three-body branching ratio and $Y_{\tilde{\nu}} M_{\tilde{\nu}}$ for another case: $m_{1 / 2}=500 \mathrm{GeV}, m_{0}=100 \mathrm{GeV}, \tan \beta=40, A_{0}=0$ and $m_{A}=1300 \mathrm{GeV}$. We see in panel (c) that the three-body branching ratio is smaller than $10^{-6}$ for $M_{\tilde{\nu}}<$ $110 \mathrm{GeV}$, and always $<3 \times 10^{-5}$. Panel (d) shows that $Y_{\tilde{\nu}} M_{\tilde{\nu}}$ is, again, always below about $10^{-11} \mathrm{GeV}$. In this case, the prominent dip due to the light $h$ resonance in sneutrino-pair annihilation, and the direct-channel $Z$ resonance is less important. This difference from the previous case is due to the larger value of $\tan \beta$.

These examples are indicative that the sneutrino LSP regions in the NUHM parameter space are generally safe from BBN constraints. We next check other possible constraints on decaying sneutrinos.

When the high-energy decay neutrinos are thermalized, their energy is transferred and converted to radiation. If the sneutrinos decay after about $z=10^{7}$ (corresponding to a lifetime of $1.8 \times 10^{7} \mathrm{~s}$ ), then the photons produced might not have a chance to thermalize, and could show up as distortion of the CMB black-body radiation spectrum. These constraints were also considered in [19] where it was found that for lifetimes between $10^{7}$ and 

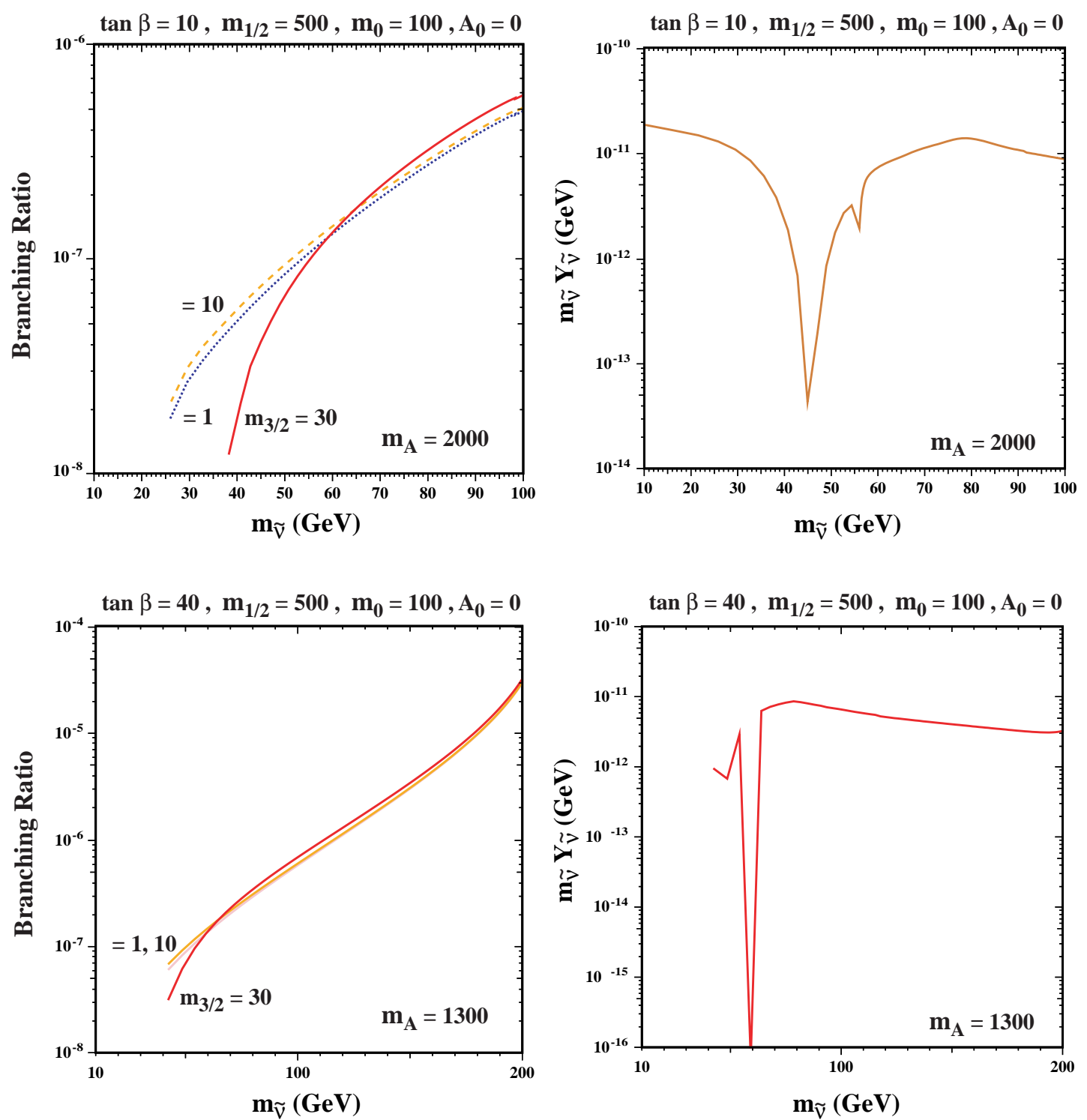

Figure 4: Exploration of the BBN constraints on sample tau-sneutrino NLSP points with $\mu>0$ : (top) $m_{1 / 2}=500 \mathrm{GeV}, m_{0}=100 \mathrm{GeV}, \tan \beta=10, A_{0}=0$ and $m_{A}=2000 \mathrm{GeV}$ [cf, figure 2(d)] and (bottom) $m_{1 / 2}=500 \mathrm{GeV}, m_{0}=100 \mathrm{GeV}, \tan \beta=40, A_{0}=0$ and $m_{A}=1300 \mathrm{GeV}$ [cf, figure 3(d)]. Panels (a, c) display the three-body-decay branching ratios, and panels $(\mathrm{b}, \mathrm{d})$ the $\tilde{\nu}_{(\tau, e)}$ relic density.

$10^{13} \mathrm{~s}$, the upper limit on $Y_{\tilde{\nu}} M_{\tilde{\nu}}$ is roughly $10^{-9}\left(10^{-7}\right) \mathrm{GeV}$ for $B_{h}=10^{-3}\left(10^{-6}\right)$. Thus in the parameter space we are interested in, this too is never a serious constraint.

When the sneutrino and gravitino mass are nearly degenerate, the sneutrino lifetime might be very long ( $\gtrsim 10^{13} \mathrm{~s}$ as seen in figure 1). If the sneutrinos have decayed after the time of recombination, they will produce a diffuse neutrino and photon background. In principle, there is then a very strong constraint from water Cerenkov detectors placing an upper limit on $Y_{\tilde{\nu}} M_{\tilde{\nu}}$ of order $10^{-12}-10^{-15} \mathrm{GeV}$ [42]. However, these detectors lose 
sensitivity for neutrino energies below about $10 \mathrm{GeV}$ [44. Thus we again do not expect a severe constraint placed on the parameter space of interest.

\section{Signatures of metastable sneutrinos at colliders}

We have seen in the previous sections that a sneutrino NLSP is a generic possibility in the NUHM. It would be metastable, so that its decays would not be seen at colliders, but the late decays of relic sneutrinos are not excluded by the available cosmological constraints. The mass of such a sneutrino NLSP might be as low as the LEP lower limit [38, 39]. As a non-decaying neutral particle, the sneutrino would have a missing-energy signature at colliders. Distinguishing the sneutrino from other possible origins of such events would require a search for the heavier states that decay into the sneutrino inside the detector.

Covi and Kraml 21] have studied several scenarios with a sneutrino NLSP assuming the following mass hierarchies. (a) $m_{\tilde{\tau}}>m_{\chi}>m_{\tilde{\nu}_{\tau}}$ : in this case, neutralino decays into a neutrino and sneutrino are invisible, and the signatures of such decay chains resemble those in a conventional neutralino LSP scenario. However, if $m_{\chi}>m_{\tilde{\nu}_{\tau}}+m_{\tau}$, the neutralino can decay into $\chi \rightarrow \tau \tilde{\nu}_{\tau} f \bar{f}^{\prime}$, where the $f \bar{f}^{\prime}$ pair is soft if the mass gap is small. (b) $m_{\chi}>m_{\tilde{\tau}}>m_{\tilde{\nu}_{\tau}}$ : in this case, besides the invisible $\nu+\widetilde{\nu}$ decay mode, the neutralino can undergo cascade decays that might be detectable, such as $\tau+\tilde{\tau}$. (c) $m_{\chi}>m_{\tilde{e}_{L}}>m_{\tilde{\tau}}>m_{\tilde{\nu}_{\tau}}$ : in this case, there are additional decay channels with neutralino decay into an electron and a selectron which subsequently decays into leptons and a tau-sneutrino. Heavier sparticles might decay via the lightest neutralino, but they might also decay directly into the $\widetilde{\nu}$, e.g., via $\chi_{2} \rightarrow \nu+\widetilde{\nu}$, or via sleptons, e.g., $\chi_{2} \rightarrow \tau+\tilde{\tau}, \tilde{\tau} \rightarrow \widetilde{\nu}+f+\bar{f}^{\prime}$.

We see from figure 2 that there are several possible scenarios for the sparticle mass spectrum in the NUHM, which are distinct from the standard CMSSM spectrum as we now enumerate.

1. $m_{\chi}>m_{\tilde{e}_{L}}>m_{\tilde{\nu}_{e}}>m_{\tilde{\tau}}>m_{\tilde{\nu}_{\tau}}$ :

This is the mass hierarchy seen in panel (d) of figure 2 for larger $m_{A}$. The neutralino can decay into

$$
\begin{aligned}
\chi \rightarrow & \tilde{e}_{L}+e \\
& \tilde{\nu}_{e}+\nu_{e} \\
& \tilde{\tau}_{1}+\tau \\
& \tilde{\nu}_{\tau}+\nu_{\tau}
\end{aligned}
$$

which, for the first mode, would be followed by

$$
\begin{aligned}
\tilde{e}_{L} \rightarrow & \tilde{\nu}_{e}+\bar{f}^{\prime}+f \\
& \tilde{\tau}_{1}+e+\tau \\
& \tilde{\tau}_{1}+\nu_{e}+\nu_{\tau}
\end{aligned}
$$




$$
\begin{aligned}
& \tilde{\nu}_{\tau}+e+\nu_{\tau} \\
& \tilde{\nu}_{\tau}+\nu_{e}+\tau,
\end{aligned}
$$

the second mode by

$$
\begin{aligned}
\tilde{\nu}_{e} \rightarrow & \tilde{\tau}_{1}+\nu_{e}+\tau \\
& \tilde{\tau}_{1}+e+\nu_{\tau} \\
& \tilde{\nu}_{\tau}+\nu_{e}+\nu_{\tau} \\
& \tilde{\nu}_{\tau}+e+\tau,
\end{aligned}
$$

and the third mode by

$$
\tilde{\tau}_{1} \rightarrow \tilde{\nu}_{\tau}+\bar{f}^{\prime}+f
$$

In the case of the two-body neutralino decay to stau and tau, the decay rate is

$$
\begin{aligned}
\Gamma\left(\chi \rightarrow \tilde{\tau}_{1}+\tau\right)= & \frac{\sqrt{m_{\chi}^{4}+m_{\tau}^{4}+m_{\tilde{\tau}}^{4}-2\left(m_{\tau}^{2} m_{\tilde{\tau}}^{2}+m_{\chi}^{2} m_{\tau}^{2}+m_{\chi}^{2} m_{\tilde{\tau}}^{2}\right)}}{32 \pi m_{\chi}^{3}} \\
& \times\left(\left(\left|C_{R}\right|^{2}+\left|C_{L}\right|^{2}\right)\left(m_{\chi}^{2}+m_{\tau}^{2}-m_{\tilde{\tau}}^{2}\right)-2\left(C_{L} C_{R}^{*}+C_{R} C_{L}^{*}\right) m_{\tau} m_{\chi}\right)
\end{aligned}
$$

where $C_{L}, C_{R}$ are the left and right couplings in the neutralino-tau-stau vertex. There are similar expressions are for the other two-body decay modes.

2. $m_{\chi}>m_{\tilde{\nu}_{\tau}}>m_{\tilde{\tau}}>m_{\tilde{e}_{L}}>m_{\tilde{\nu}_{e}}:$

This hierarchy occurs for more intermediate values of $m_{A}$ when $|\mu|$ is large as seen in panels (b) and (c) of figure 2 ${ }^{14}$ The neutralino 2-body decay modes would be the same as in the previous case, although with different branching ratios. However, the cascade decays are in general different. In this case, we would have

$$
\begin{aligned}
& \tilde{\nu}_{\tau} \rightarrow \tilde{\tau}_{1}+\bar{f}^{\prime}+f \\
& \tilde{e}_{L}+e+\nu_{\tau} \\
& \tilde{e}_{L}+\nu_{e}+\tau \\
& \tilde{\nu}_{e}+\nu_{e}+\nu_{\tau} \\
& \tilde{\nu}_{\tau}+e+\tau \\
& \\
& \tilde{\tau} \rightarrow \tilde{e}_{L}+e+\tau \\
& \tilde{e}_{L}+\nu_{e}+\nu_{\tau} \\
& \tilde{\nu}_{e}+\nu_{e}+\tau \\
& \tilde{\nu}_{e}+e+\nu_{\tau}
\end{aligned}
$$

and

$$
\tilde{e}_{L} \rightarrow \tilde{\nu}_{e}+\bar{f}^{\prime}+f
$$

\footnotetext{
${ }^{14}$ The viability of such models would require some action to conform with the GUT constraint, e.g., by constraining inflationary cosmology.
} 
3. For large $\tan \beta$, e.g. $\tan \beta=40$ as shown in panel $\mathrm{d}$ of figure 3 , and large $|\mu|$, the third-generation sleptons get larger masses through the Yukawa couplings. Thus we have $m_{\chi}>m_{\tilde{e}_{L}}>m_{\tilde{\nu}_{e}}$ for the lightest sparticles. In this case, the neutralino cascade decays become simpler,

$$
\begin{aligned}
\chi \rightarrow & \tilde{e}_{L}+e \\
& \tilde{\nu}_{e}+\nu_{e}
\end{aligned}
$$

which, for the first mode, would be followed by

$$
\tilde{e}_{L} \rightarrow \tilde{\nu}_{e}+\bar{f}^{\prime}+f
$$

whilst the second mode is invisible.

4. There are also other possibilities for narrower region of parameter space, near where the masses cross each other in figure 国: (a) $m_{\tilde{\nu}_{\tau}}>m_{\tilde{\tau}}>m_{\tilde{e}_{L}}>m_{\chi}>m_{\tilde{\nu}_{e}}$; (b) $m_{\tilde{\nu}_{\tau}}>m_{\tilde{\tau}}>m_{\chi}>m_{\tilde{e}_{L}}>m_{\tilde{\nu}_{e}} ;$ and (c) $m_{\tilde{\nu}_{\tau}}>m_{\chi}>m_{\tilde{\tau}}>m_{\tilde{e}_{L}}>m_{\tilde{\nu}_{e}}$.

We extract from these examples a few generic features. As in the cases of many other scenarios beyond the Standard Model, particularly within the general framework of supersymmetry, the most prominent signature of a sneutrino NLSP scenario is likely to be missing energy. However, there would in general be accompanying signatures that would enable a sneutrino NLSP scenario to be distinguished from other possibilities. Specifically, one expects to see also events with missing energy accompanied by leptons. The precise nature of this supplementary signature would, however, depend on the nature of the sneutrino: $\tilde{\nu}_{\tau}, \tilde{\nu}_{\mu}$ or $\tilde{\nu}_{e}$, and on the hierarchy of heavier sparticle masses.

In particular, the relative multiplicities of different charged leptons would depend on the flavour of the invisible $\widetilde{\nu}$, and hence be a useful tool for identifying it. Concretely, in cases where the parent sparticle has no lepton flavour, as would normally be the case at the LHC, each $\tilde{\nu}_{\tau}$ NLSP would be accompanied by an unmatched $\tau$ or $\nu_{\tau}$, and each $\tilde{\nu}_{e, \mu}$ NLSP would be accompanied by an unmatched electron, $\mu$ or corresponding neutrino. In general, there would be additional lepton-antilepton pairs with matched flavours.

\section{Summary}

We have analyzed in this paper the possibility of a sneutrino NLSP in NUHM models with a gravitino LSP. This possibility does not exist in the CMSSM, but is quite generic in the NUHM, as we have illustrated with various specific examples. The sneutrino might well be the $\tilde{\nu}_{\tau}$, but the $\tilde{\nu}_{\mu}$ and $\tilde{\nu}_{e}$ are also possible candidates for the NLSP. A sneutrino NLSP would be metastable and subject to cosmological constraints on late-decaying particles, but we have shown that these are not difficult to respect. There are various different possible scenarios for the spectrum of sparticles heavier than the sneutrino, which would have distinctive signatures at colliders. In addition to events with missing energy carried 
away by the invisible $\widetilde{\nu}$, there would also be events with accompanying charged leptons. The flavours of such leptons would help identify the flavour of the sneutrino NLSP. ${ }^{15}$

As particle physics embarks on the study of the TeV scale with the LHC, much unknown physics will surely be revealed. Supersymmetry is occasionally regarded as a 'known unknown' in the sense that, whereas we do not know whether it exists, we think we know what it would look like if it does exist. This paper reminds us that supersymmetry should rather be regarded as an 'unknown unknown', in the sense that not only do we not know whether it exists, but we also do not know what it would look like. In the conventional 'known unknown' scenario, the LSP is the lightest neutralino and supersymmetry would produce missing-energy events. The latter would also be the signature of a scenario with a gravitino LSP with a neutralino NLSP, at least in gravity-mediated scenarios. However, once the 'Pandora's box' of a gravitino LSP has been opened, many other NLSP candidates fly out. In addition to the relatively familiar case of the lighter stau and the more radical case of the lighter stop, there are other possibilities including the sneutrino NLSP scenarios discussed here. All of these scenarios have distinctive features, as illustrated here, so the LHC and subsequent experiments have good prospects for detecting and distinguishing between the various 'unknown unknowns'. No 'unknown unknown' stone should be left unturned in the search for supersymmetry.

\section{Acknowledgments}

The work of K.A.O. was supported in part by DOE grant DE-FG02-94ER-40823. Y.S. is grateful for the hospitality of CERN and University of Victoria where part of this work was done, and thanks Terrance Figy, Gudrun Heinrich, Stefan Hesselbach, Emerson Luna and Kazunori Kohri for useful discussions.

\section{A. Sneutrino three-body decay}

We calculate here the radiative sneutrino three-body decay

$$
\tilde{\nu}(P) \rightarrow \nu(p)+\gamma^{\sigma}(k)+\widetilde{G}^{\mu}\left(P_{G}\right)
$$

that may arise through the photino content of the neutralino, as illustrated in the diagram below.

\footnotetext{
${ }^{15}$ Note added: There was recent announcement from CDF [45], after this paper was completed, that put a stronger constraint on the lower bound of the chargino mass from the trilepton signal search at the Tevatron. In our model, the chargino is generally heavier compared to that in the neutralino LSP scenario assumed in the CDF analysis. In addition, the cross sections might also be different. Therefore this bound cannot be applied directly to our models.
} 


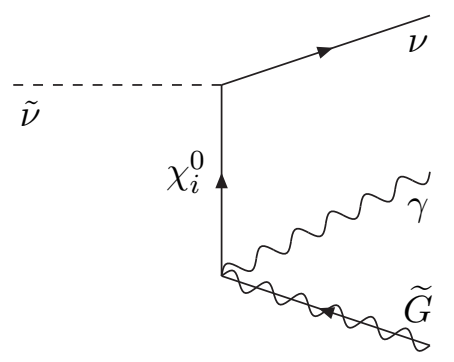

The invariant amplitude for this decay is

$$
\mathcal{M}=\frac{-i B_{i} C_{i}}{4 M_{\mathrm{Pl}}\left(q^{2}-m_{i}^{2}\right)} \bar{u}(p) P_{R}\left(\not q+m_{i}\right) \gamma_{\mu}\left[\not h, \gamma_{\sigma}\right] \Psi^{\mu}\left(P_{G}\right) \epsilon^{\sigma}(k)
$$

where $M_{\mathrm{Pl}}=1 / \sqrt{8 \pi G_{N}}$ is the Planck mass, $m_{i} \equiv m_{\chi_{i}^{0}}$,

$$
q \equiv k+P_{G}
$$

and the dimensionless couplings are

$$
\begin{aligned}
B_{i} & \equiv-\frac{g_{2}}{\sqrt{2}}\left(O_{2 i}-\tan \theta_{W} O_{1 i}\right) \\
C_{i} & \equiv O_{1 i} \cos \theta_{W}+O_{2 i} \sin \theta_{W} .
\end{aligned}
$$

Ignoring the neutrino mass, we get

$$
\begin{aligned}
\overline{|\mathcal{M}|^{2}}= & \frac{8}{3} \frac{k \cdot P_{G}}{M_{\mathrm{Pl}}^{2}} \sum_{i, j} \frac{C_{j}^{*} C_{i} B_{j}^{*} B_{i}}{\left(q^{2}-m_{i}^{2}\right)\left(q^{2}-m_{j}^{2}\right)} \\
= & \left\{\frac{k \cdot P_{G}}{m_{G}^{2}}\left[2\left(P_{G} \cdot q\right)(p \cdot q)+\left(P_{G} \cdot p\right)\left(m_{i} m_{j}-q^{2}\right)\right]\right. \\
& \left.\quad+2(k \cdot q)(p \cdot q)+(k \cdot p)\left(m_{i} m_{j}-q^{2}\right)\right\} .
\end{aligned}
$$

Use the Dalitz parametrization $m_{12} \equiv p_{1}+p_{2}, m_{23} \equiv p_{2}+p_{3}$ with $p_{1}=P_{G}, p_{2}=k, p_{3}=p$, we get

$$
\begin{aligned}
P_{G} \cdot k & =\frac{1}{2}\left(m_{12}^{2}-m_{G}^{2}\right) \\
k \cdot p & =\frac{1}{2} m_{23}^{2} \\
P_{G} \cdot p & =\frac{1}{2}\left(M^{2}-m_{12}^{2}-m_{23}^{2}\right) \\
k \cdot q & =\frac{1}{2}\left(m_{12}^{2}-m_{G}^{2}\right) \\
p \cdot q & =\frac{1}{2}\left(M^{2}-m_{12}^{2}\right) \\
P_{G} \cdot q & =\frac{1}{2}\left(m_{12}^{2}+m_{G}^{2}\right)
\end{aligned}
$$

and

$$
q^{2}=m_{12}^{2}
$$

Hereafter, we abbreviate our notation by defining $M \equiv m_{\tilde{\nu}}$ and $m_{G} \equiv m_{\widetilde{G}}$. 
The resulting partial decay rate is [39]

$$
d \Gamma=\frac{1}{(2 \pi)^{3}} \frac{1}{32 M^{3}} \overline{|\mathcal{M}|^{2}} d m_{12}^{2} d m_{23}^{2} .
$$

This can be integrated analytically using the following integration boundaries - for $m_{23}^{2}$ : 0 and $\left(m_{12}^{2} M^{2}+m_{12}^{2} m_{G}^{2}-m_{12}^{4}-M^{2} m_{G}^{2}\right) / m_{12}^{2}$, and for $m_{12}^{2}: m_{G}^{2}$ to $M^{2}$. The result is

$$
\Gamma=\frac{1}{768 \pi^{3} M_{\mathrm{Pl}}^{2} M^{3}} \sum_{i, j} \frac{C_{j}^{*} C_{i} B_{j}^{*} B_{i}}{2}\left(I_{a}(i, j)+I_{b}(i, j)+I_{c}(i, j)+I_{d}(i, j)\right),
$$

where

$$
\begin{gathered}
I_{a}(i, j) \equiv \frac{m_{i}^{2}+m_{j}^{2}}{m_{G}^{2}\left(m_{i} m_{j}\right)^{4}}\left[\sum_{a=1}^{7} \frac{\alpha_{a}(i, j)}{a}\left(M^{2 a}-m_{G}^{2 a}\right)+\alpha_{0}(i, j) \ln \left[\frac{M^{2}}{m_{G}^{2}}\right]\right], \\
I_{b}(i, j) \equiv \frac{1}{m_{G}^{2}\left(m_{i} m_{j}\right)^{2}}\left[\sum_{a=1}^{6} \frac{\alpha_{a+1}(i, j)}{a}\left(M^{2 a}-m_{G}^{2 a}\right)+\alpha_{1}(i, j) \ln \left[\frac{M^{2}}{m_{G}^{2}}\right]\right. \\
\left.-\alpha_{0}(i, j)\left(\frac{1}{M^{2}}-\frac{1}{m_{G}^{2}}\right)\right], \\
I_{c}(i, j) \equiv \frac{1}{m_{G}^{2} m_{i}^{4}\left(m_{i}^{2}-m_{j}^{2}\right)}\left[\sum_{a=1}^{7} \frac{\beta_{a}(i, j)}{a}\left(\left(M^{2}-m_{i}^{2}\right)^{a}-\left(m_{G}^{2}-m_{i}^{2}\right)^{a}\right)\right. \\
\left.+\beta_{0}(i, j) \ln \left[\frac{M^{2}-m_{i}^{2}}{m_{G}^{2}-m_{i}^{2}}\right]\right],
\end{gathered}
$$

and

$$
I_{d}(i, j) \equiv I_{c}(j, i)
$$

where the auxiliary functions $\alpha$ and $\beta$ are defined below. Note that there is no actual singularity when $i=j$, because $I_{c}+I_{d}$ is of the form

$$
\left(\frac{f(a, b)}{a^{2}}-\frac{f(b, a)}{b^{2}}\right) \frac{1}{a-b}
$$

and in this case, with $m_{i}=m_{j}$, we get

$$
\begin{aligned}
I_{c}+I_{d}= & \frac{1}{m_{G}^{2} m_{i}^{2}}\left[-2 \frac{u^{7}}{7}+\left(-13 m_{i}^{2}-2 \alpha_{6}\right) \frac{u^{6}}{6}+\left(-35 m_{i}^{4}-11 m_{i}^{2} \alpha_{6}-2 \alpha_{5}\right) \frac{u^{5}}{5}\right. \\
& +\left(-49 m_{i}^{6}-24 m_{i}^{4} \alpha_{6}-9 m_{i}^{2} \alpha_{5}-2 \alpha_{4}\right) \frac{u^{4}}{4} \\
& +\left(-35 m_{i}^{8}-25 m_{i}^{6} \alpha_{6}-15 m_{i}^{4} \alpha_{5}-7 m_{i}^{2} \alpha_{4}-2 \alpha_{3}\right) \frac{u^{3}}{3} \\
& +\left(-7 m_{i}^{10}-10 m_{i}^{8} \alpha_{6}-10 m_{i}^{6} \alpha_{5}-8 m_{i}^{4} \alpha_{4}-5 m_{i}^{2} \alpha_{3}-2 \alpha_{2}\right) \frac{u^{2}}{2} \\
& +\left(7 m_{i}^{12}+3 m_{i}^{10} \alpha_{6}-2 m_{i}^{6} \alpha_{4}-3 m_{i}^{4} \alpha_{3}-3 m_{i}^{2} \alpha_{2}-2 \alpha_{1}\right) u \\
& +\left(5 m_{i}^{14}+4 m_{i}^{12} \alpha_{6}+3 m_{i}^{10} \alpha_{5}+2 m_{i}^{8} \alpha_{4}+m_{i}^{6} \alpha_{3}-m_{i}^{2} \alpha_{1}-2 \alpha_{0}\right) \ln u \\
& \left.-\left(m_{i}^{16}+m_{i}^{14} \alpha_{6}+m_{i}^{12} \alpha_{5}+m_{i}^{10} \alpha_{4}+m_{i}^{8} \alpha_{3}+m_{i}^{6} \alpha_{2}+m_{i}^{4} \alpha_{1}+m_{i}^{2} \alpha_{0}\right) \frac{1}{u}\right]_{u=m_{G}^{2}-m_{i}^{2}}^{M^{2}-m_{i}^{2}}(\mathrm{~A} .21)
\end{aligned}
$$


The auxiliary functions are

$$
\begin{aligned}
& \alpha_{0}(i, j) \equiv-3 m_{i} m_{j} m_{G}^{8} M^{4} \\
& \alpha_{1}(i, j) \equiv m_{i} m_{j}\left(8 m_{G}^{6} M^{4}+6 M^{2} m_{G}^{8}\right)-3 m_{G}^{8} M^{4} \\
& \alpha_{2}(i, j) \equiv 8 m_{G}^{6} M^{4}+6 M^{2} m_{G}^{8}-m_{i} m_{j}\left(3 m_{G}^{8}+16 M^{2} m_{G}^{6}+6 M^{4} m_{G}^{4}\right) \\
& \alpha_{3}(i, j) \equiv m_{i} m_{j}\left(8 m_{G}^{6}+12 M^{2} m_{G}^{4}\right)-3 m_{G}^{8}-16 M^{2} m_{G}^{6}-6 M^{4} m_{G}^{4} \\
& \alpha_{4}(i, j) \equiv m_{i} m_{j}\left(M^{4}-6 m_{G}^{4}\right)+8 m_{G}^{6}+12 M^{2} m_{G}^{4} \\
& \alpha_{5}(i, j) \equiv M^{4}-6 m_{G}^{4}-2 m_{i} m_{j} M^{2} \\
& \alpha_{6}(i, j) \equiv m_{i} m_{j}-2 M^{2} \\
& \alpha_{7}(i, j) \equiv 1
\end{aligned}
$$

and

$$
\begin{aligned}
\beta_{0}(i, j) & \equiv \sum_{a=0}^{7} m_{i}^{2 a} \alpha_{a}(i, j) \\
\beta_{1}(i, j) & \equiv \sum_{a=1}^{7} a m_{i}^{2(a-1)} \alpha_{a}(i, j) \\
\beta_{2}(i, j) & \equiv 21 m_{i}^{10}+15 m_{i}^{8} \alpha_{6}+10 m_{i}^{6} \alpha_{5}+6 m_{i}^{4} \alpha^{4}+3 m_{i}^{2} \alpha_{3}+\alpha_{2} \\
\beta_{3}(i, j) & \equiv 35 m_{i}^{8}+20 m_{i}^{6} \alpha_{6}+10 m_{i}^{4} \alpha_{5}+4 m_{i}^{2} \alpha_{4}+\alpha_{3} \\
\beta_{4}(i, j) & \equiv 35 m_{i}^{6}+15 m_{i}^{4} \alpha_{6}+5 m_{i}^{2} \alpha_{5}+\alpha_{4} \\
\beta_{5}(i, j) & \equiv 21 m_{i}^{4}+6 m_{i}^{2} \alpha_{6}+\alpha_{5} \\
\beta_{6}(i, j) & \equiv 7 m_{i}^{2}+\alpha_{6} \\
\beta_{7}(i, j) & \equiv 1 .
\end{aligned}
$$

\section{References}

[1] J. Rich, D. Lloyd Owen and M. Spiro, Experimental particle physics without accelerators, Phys. Rept. 151 (1987) 239;

P.F. Smith, Terrestrial searches for new stable particles, Contemp. Phys. 29 (1998) 159;

T.K. Hemmick et al., A search for anomalously heavy isotopes of low Z nuclei, Phys. Rev. D 41 (1990) 2074;

C.F. Berger, L. Covi, S. Kraml and F. Palorini, The number density of a charged relic, arXiv:0807.0211.

[2] T. Falk, K.A. Olive and M. Srednicki, Heavy sneutrinos as dark matter, Phys. Lett. B 339 (1994) 248 hep-ph/9409270.

[3] J.R. Ellis, J.S. Hagelin, D.V. Nanopoulos, K.A. Olive and M. Srednicki, Supersymmetric relics from the big bang, Nucl. Phys. B 238 (1984) 453;

H. Goldberg, Constraint on the photino mass from cosmology, Phys. Rev. Lett. 50 (1983) 1419 .

[4] J.R. Ellis, K.A. Olive, Y. Santoso and V.C. Spanos, Gravitino dark matter in the CMSSM, Phys. Lett. B 588 (2004) 7 hep-ph/0312262. 
[5] J.L. Feng, A. Rajaraman and F. Takayama, Superweakly-interacting massive particles, Phys. Rev. Lett. 91 (2003) 011302 hep-ph/0302215]; SuperWIMP dark matter signals from the early universe, Phys. Rev. D 68 (2003) 063504 hep-ph/0306024;

J.L. Feng, S.-F. Su and F. Takayama, Supergravity with a gravitino LSP, Phys. Rev. D 70 (2004) 075019 hep-ph/0404231.

[6] J.L. Feng, S.-F. Su and F. Takayama, SuperWIMP gravitino dark matter from slepton and sneutrino decays, Phys. Rev. D 70 (2004) 063514 hep-ph/0404198.

[7] J.R. Ellis, K.A. Olive, Y. Santoso and V.C. Spanos, Supersymmetric dark matter in light of WMAP, Phys. Lett. B 565 (2003) 176 hep-ph/0303043.

[8] H. Baer and C. Balázs, $\chi^{2}$ analysis of the minimal supergravity model including WMAP, $g_{\mu}-2$ and $b \rightarrow s \gamma$ constraints, JCAP 05 (2003) 006 hep-ph/0303114;

A.B. Lahanas and D.V. Nanopoulos, WMAPing out supersymmetric dark matter and phenomenology, Phys. Lett. B 568 (2003) 55 hep-ph/0303130;

U. Chattopadhyay, A. Corsetti and P. Nath, WMAP constraints, SUSY dark matter and implications for the direct detection of SUSY, Phys. Rev. D 68 (2003) 035005

hep-ph/0303201;

C. Muñoz, Dark matter detection in the light of recent experimental results, Int. J. Mod. Phys. A 19 (2004) 3093 hep-ph/0309346.

[9] J.R. Ellis, K.A. Olive, Y. Santoso and V.C. Spanos, Very constrained minimal supersymmetric standard models, Phys. Rev. D 70 (2004) 055005 hep-ph/0405110.

[10] D. Matalliotakis and H.P. Nilles, Implications of nonuniversality of soft terms in supersymmetric grand unified theories, Nucl. Phys. B 435 (1995) 115 hep-ph/9407251;

M. Olechowski and S. Pokorski, Electroweak symmetry breaking with nonuniversal scalar soft terms and large tan beta solutions, Phys. Lett. B 344 (1995) 201 hep-ph/9407404;

V. Berezinsky et al., Neutralino dark matter in supersymmetric models with nonuniversal scalar mass terms, Astropart. Phys. 5 (1996) 1 hep-ph/9508249;

M. Drees, M.M. Nojiri, D.P. Roy and Y. Yamada, Light Higgsino dark matter, Phys. Rev. D 56 (1997) 276 [Erratum ibid. D 64 (1997) 039901] hep-ph/9701219];

M. Drees et al., Scrutinizing LSP dark matter at the LHC, Phys. Rev. D 63 (2001) 035008 hep-ph/0007202;

P. Nath and R.L. Arnowitt, Non-universal soft SUSY breaking and dark matter, Phys. Rev. D 56 (1997) 2820 hep-ph/9701301;

J.R. Ellis, T. Falk, G. Ganis, K.A. Olive and M. Schmitt, Charginos and neutralinos in the light of radiative corrections: sealing the fate of Higgsino dark matter, Phys. Rev. D 58 (1998) 095002 hep-ph/9801445;

J.R. Ellis, T. Falk, G. Ganis and K.A. Olive, Supersymmetric dark matter in the light of LEP and the Tevatron collider, Phys. Rev. D 62 (2000) 075010 hep-ph/0004169;

A. Bottino, F. Donato, N. Fornengo and S. Scopel, Probing the supersymmetric parameter space by WIMP direct detection, Phys. Rev. D 63 (2001) 125003 hep-ph/0010203;

S. Profumo, Neutralino dark matter, b- $\tau$ Yukawa unification and non-universal sfermion masses, Phys. Rev. D 68 (2003) 015006 hep-ph/0304071;

D.G. Cerdeno and C. Muñoz, Neutralino dark matter in supergravity theories with nonuniversal scalar and gaugino masses, JHEP 10 (2004) 015 hep-ph/0405057.

[11] J.R. Ellis, K.A. Olive and Y. Santoso, The MSSM parameter space with non-universal Higgs masses, Phys. Lett. B 539 (2002) 107 hep-ph/0204192. 
[12] J.R. Ellis, T. Falk, K.A. Olive and Y. Santoso, Exploration of the MSSM with non-universal Higgs masses, Nucl. Phys. B 652 (2003) 259 hep-ph/0210205.

[13] H. Baer, A. Mustafayev, S. Profumo, A. Belyaev and X. Tata, Direct, indirect and collider detection of neutralino dark matter in SUSY models with non-universal Higgs masses, JHEP 07 (2005) 065 hep-ph/0504001.

[14] J. Ellis, K.A. Olive and P. Sandick, Varying the universality of supersymmetry-breaking contributions to MSSM Higgs boson masses, arXiv:0805.2343.

[15] J.L. Diaz-Cruz, J.R. Ellis, K.A. Olive and Y. Santoso, On the feasibility of a stop NLSP in gravitino dark matter scenarios, JHEP 05 (2007) 003 hep-ph/0701229.

[16] W. Buchmüller, L. Covi, J. Kersten and K. Schmidt-Hoberg, Dark matter from gaugino mediation, JCAP 11 (2006) 007 hep-ph/0609142.

[17] R.H. Cyburt, J.R. Ellis, B.D. Fields and K.A. Olive, Updated nucleosynthesis constraints on unstable relic particles, Phys. Rev. D 67 (2003) 103521 astro-ph/0211258;

M. Kawasaki, K. Kohri and T. Moroi, Hadronic decay of late-decaying particles and big-bang nucleosynthesis, Phys. Lett. B 625 (2005) 7 astro-ph/0402490]; Big-bang nucleosynthesis and hadronic decay of long-lived massive particles, Phys. Rev. D 71 (2005) 083502 astro-ph/0408426;

J.R. Ellis, K.A. Olive and E. Vangioni, Effects of unstable particles on light-element abundances: lithium versus deuterium and He 3 , Phys. Lett. B 619 (2005) 30 astro-ph/0503023;

K. Kohri, T. Moroi and A. Yotsuyanagi, Big-bang nucleosynthesis with unstable gravitino and upper bound on the reheating temperature, Phys. Rev. D 73 (2006) 123511 hep-ph/0507245;

D.G. Cerdeno, K.-Y. Choi, K. Jedamzik, L. Roszkowski and R. Ruiz de Austri, Gravitino dark matter in the CMSSM with improved constraints from BBN, JCAP 06 (2006) 005 hep-ph/0509275;

F.D. Steffen, Gravitino dark matter and cosmological constraints, JCAP 09 (2006) 001 hep-ph/0605306.

[18] R.H. Cyburt, J.R. Ellis, B.D. Fields, K.A. Olive and V.C. Spanos, Bound-state effects on light-element abundances in gravitino dark matter scenarios, JCAP 11 (2006) 014 astro-ph/0608562.

[19] T. Kanzaki, M. Kawasaki, K. Kohri and T. Moroi, Cosmological constraints on neutrino injection, Phys. Rev. D 76 (2007) 105017 arXiv:0705.1200].

[20] T. Kanzaki, M. Kawasaki, K. Kohri and T. Moroi, Cosmological constraints on gravitino LSP scenario with sneutrino NLSP, Phys. Rev. D 75 (2007) 025011 hep-ph/0609246.

[21] L. Covi and S. Kraml, Collider signatures of gravitino dark matter with a sneutrino NLSP, JHEP 08 (2007) 015 hep-ph/0703130.

[22] V. Barger, D. Marfatia and A. Mustafayev, Neutrino sector impacts SUSY dark matter, Phys. Lett. B 665 (2008) 242 arXiv:0804.3601.

[23] S.P. Martin and M.T. Vaughn, Two loop renormalization group equations for soft supersymmetry breaking couplings, Phys. Rev. D 50 (1994) 2282 [Erratum ibid. D 78 (2008) 039903] hep-ph/9311340].

[24] V.D. Barger, M.S. Berger and P. Ohmann, The supersymmetric particle spectrum, Phys. Rev. D 49 (1994) 4908 hep-ph/9311269. 
[25] W. de Boer, R. Ehret and D.I. Kazakov, Predictions of SUSY masses in the minimal supersymmetric grand unified theory, Z. Physik C 67 (1995) 647 hep-ph/9405342.

[26] H. Baer and X. Tata, Weak scale supersymmetry: from superfields to scattering events, Cambridge University Press, Cambridge U.K. (2006).

[27] P. Binetruy, Supersymmetry: theory, experiment and cosmology, Oxford University Press, Oxford U.K. (2006).

[28] M.S. Carena, J.R. Ellis, A. Pilaftsis and C.E.M. Wagner, Higgs-boson pole masses in the MSSM with explicit CP-violation, Nucl. Phys. B 625 (2002) 345 hep-ph/0111245.

[29] L.E. Ibáñez, C. Lopez and C. Muñoz, The low-energy supersymmetric spectrum according to $N=1$ supergravity guts, Nucl. Phys. B 256 (1985) 218.

[30] J.R. Ellis, K.A. Olive, Y. Santoso and V.C. Spanos, High-energy constraints on the direct detection of MSSM neutralinos, Phys. Rev. D 69 (2004) 015005 hep-ph/0308075.

[31] T. Falk, K.A. Olive, L. Roszkowski and M. Srednicki, New constraints on superpartner masses, Phys. Lett. B 367 (1996) 183 hep-ph/9510308.

[32] A. Riotto and E. Roulet, Vacuum decay along supersymmetric flat directions, Phys. Lett. B 377 (1996) 60 hep-ph/9512401;

A. Kusenko, P. Langacker and G. Segre, Phase transitions and vacuum tunneling into charge and color breaking minima in the MSSM, Phys. Rev. D 54 (1996) 5824 hep-ph/9602414;

T. Falk, K.A. Olive, L. Roszkowski, A. Singh and M. Srednicki, Constraints from inflation and reheating on superpartner masses, Phys. Lett. B 396 (1997) 50 hep-ph/9611325.

[33] J. Ellis, J. Giedt, O. Lebedev, K. Olive and M. Srednicki, Against tachyophobia, arXiv:0806.3648.

[34] WMAP collaboration, J. Dunkley et al., Five-year Wilkinson Microwave Anisotropy Probe (WMAP) observations: likelihoods and parameters from the WMAP data, arXiv:0803.0586.

[35] CDF collaboration, A combination of CDF and D0 results on the mass of the top quark, arXiv:0803.1683.

[36] S. Heinemeyer, W. Hollik and G. Weiglein, FeynHiggs: a program for the calculation of the masses of the neutral CP-even Higgs bosons in the MSSM, Comput. Phys. Commun. 124 (2000) 76 hep-ph/9812320; The masses of the neutral CP-even Higgs bosons in the MSSM: accurate analysis at the two-loop level, Eur. Phys. J. C 9 (1999) 343 hep-ph/9812472.

[37] M. Davier, The hadronic contribution to $(g-2)_{\mu}$, Nucl. Phys. 169 (Proc. Suppl.) (2007) 288 hep-ph/0701163.

[38] J.R. Ellis, T. Falk, K.A. Olive and M. Schmitt, Supersymmetric dark matter in the light of LEP 1.5, Phys. Lett. B 388 (1996) 97 hep-ph/9607292.

[39] Particle Data Group collaboration, W.M. Yao et al., Review of particle physics, J. Phys. G 33 (2006) 1.

[40] J.R. Ellis, K.A. Olive, Y. Santoso and V.C. Spanos, On $B_{s} \rightarrow \mu^{+} \mu^{-}$and cold dark matter scattering in the MSSM with non-universal Higgs masses, JHEP 05 (2006) 063 hep-ph/0603136.

[41] Y. Gong and X. Chen, Cosmological constraints on invisible decay of dark matter, Phys. Rev. D 77 (2008) 103511 arXiv:0802.2296. 
[42] P. Gondolo, G. Gelmini and S. Sarkar, Cosmic neutrinos from unstable relic particles, Nucl. Phys. B 392 (1993) 111 hep-ph/9209236.

[43] M. Kawasaki and T. Moroi, Gravitino decay into a neutrino and a sneutrino in the inflationary universe, Phys. Lett. B 346 (1995) 27 hep-ph/9408321.

[44] F. Halzen and D. Hooper, High-energy neutrino astronomy: the cosmic ray connection, Rept. Prog. Phys. 65 (2002) 1025 astro-ph/0204527.

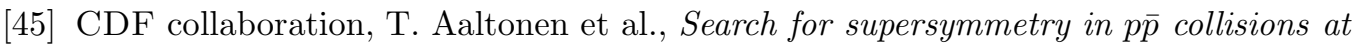
$\sqrt{s}=1.96 \mathrm{TeV}$ using the trilepton signature of chargino-neutralino production, arXiv:0808.2446. 